\title{
Editorial
}

\section{Hypotension in the Newborn: Who Needs Hydrocortisone?}

\author{
Susan W. Aucott, MD \\ Journal of Perinatology (2005) 25, 77-78. doi:10.1038/sj.jp.7211225
}

Hydrocortisone for the treatment of hypotension in newborns has become increasingly common in many neonatal intensive care units. Steroid therapy is known to increase blood pressure at all ages, but the type of steroid, the dosing regimen, and the length of treatment used varies widely. Cortisol serves to maintain vascular responsiveness to circulating vasoconstrictors. Additionally, cortisol suppresses the inflammatory response, and opposes the increased capillary permeability that occurs during acute inflammation. Individuals with adrenal insufficiency, who are unable to produce adequate cortisol, present with cardiovascular insufficiency and shock. In acutely ill adults and children, cardiovascular instability and increased mortality have been found in those patients who have normal cortisol levels, but minimal response to an adrenocorticotrophic hormone (ACTH) stimulation test. This association has been described as relative adrenal insufficiency, and responds to treatment with hydrocortisone at physiologic stress replacement doses. ${ }^{1}$

Cardiovascular instability and hypotension are common complications in sick newborns. The goal of treatment of hypotension is focused on the prevention of tissue hypoperfusion and its resultant end organ damage. In vulnerable preterm infants, cardiovascular instability can additionally lead to intraventricular hemorrhage and periventricular leukomalacia. Tissue perfusion, however, is difficult to measure directly, and the treatment is based on population-based norms for blood pressure based on gestational age. The underlying causes of hypotension are also varied, and range from intravascular volume depletion to myocardial dysfunction and lack of peripheral vasoconstriction, and may or may not include a component of relative adrenal insufficiency.

In this month's journal, two articles address the issue of hydrocortisone treatment of hypotension. The article by Fernandez et al. examines the treatment of vasopressor-resistant hypotension with hydrocortisone in term and near-term infants. Cortisol levels were obtained in infants with persistent cardiovascular instability despite management with fluid boluses and vasopressors. Response

Division of Neonatology, Johns Hopkins University, Baltimore, MD, USA.

Address correspondence and reprint requests to Susan W Aucott, MD, Division of Neonatology, Johns Hopkins University, $600 \mathrm{~N}$. Wolfe Street, Nelson 2-133, Baltimore, MD 21287, USA. to hydrocortisone treatment was evaluated based on cortisol levels being above or below $15 \mathrm{mcg} / \mathrm{dl}$. This cutoff was based on the expectation that a sick newborn should respond by producing stress levels of cortisol, thus suggesting those infants had relative adrenal insufficiency. Those infants with low cortisol levels were much more responsive to hydrocortisone therapy, as evidenced by the ability to wean vasopressor therapy, the need for fewer fluid boluses, and a decrease in heart rate. The question remains as to what is an appropriate stress cortisol level in a sick newborn. Clearly, in the setting of pressor-resistant hypotension, low to normal cortisol suggests a potentially greater benefit from hydrocortisone therapy. The converse, however, does not hold true. An appropriate stress level of cortisol in the same clinical setting may be a reflection of the varied underlying pathophysiology of hypotension, but does not predict a response to hydrocortisone therapy.

Preterm infants with cardiovascular instability present a more difficult clinical dilemma. Superimposed on the question of relative adrenal insufficiency in sick preterm infants is the developmental immaturity of the hypothalamic pituitary adrenal axis. ${ }^{2}$ Preterm infants who are not acutely ill may have low basal levels of cortisol without apparent compromise, ${ }^{3}$ making it less clear what a "normal" cortisol level should be. Studies have confirmed that hydrocortisone is beneficial for treating vasopressor-resistant hypotension, ${ }^{4,5}$ but generally are unable to correlate the need for hydrocortisone treatment with specific cortisol levels. In the current journal article by Efird et al., the use of hydrocortisone therapy in the first week of life to prevent the need for vasopressor agents was examined in extremely low birth weight infants. They found a decrease in vasopressor use on day 2 of life in the treated infants compared to controls. There was no difference in cortisol levels between the groups, and baseline cortisol levels did not correlate with the need for vasopressor use.

$\mathrm{Ng}$ et al. ${ }^{6}$ have recently examined the incidence of relative adrenal insufficiency in very low birth weight infants and its association with hypotension. Infants were enrolled prospectively and underwent human corticotrophin-releasing hormone stimulation tests at days 7 and 14 of life. Basal and peak levels were significantly associated with the lowest systolic, mean and diastolic blood pressures in the first 14 days of life, and were negatively correlated with maximum and total cumulative doses of vasopressors. No correlation of blood pressure or use of vasopressors was found with plasma ACTH on days 7 and 14, or with cortisol levels on day 14. Cortisol levels on day 7 were negatively correlated with the severity of illness scores, duration of mechanical ventilation, length of oxygen support and length of hospital stay. 
It is important to note that the basal and peak cortisol levels on day 7 were both predictive of blood pressure outcomes, indicating that a basal cortisol level may be an adequate measure, alleviating the need to perform a stimulation test in a sick infant. Levels on day 14 were not predictive, suggesting a recovery of adrenal function. Additionally, Ng notes that the serum cortisol levels on day 7 had high specificity and positive predictive value, but low sensitivity and negative predictive value for diagnosing early neonatal hypotension. This correlates with what has been documented clinically: infants with low cortisol levels with hypotension are more responsive to hydrocortisone therapy; however, there are many infants with low cortisol levels who are well, and do not go on to become hypotensive.

Defining normal levels of cortisol in sick infants remains difficult. Clearly, in symptomatic infants, low cortisol levels may indicate responsiveness to hydrocortisone therapy. Conversely, high levels of cortisol are often signs of excessive pain and stress and have been correlated with increased morbidity and mortality. ${ }^{7}$ The question remains as to what cortisol level is appropriate: too high may be detrimental, and yet it must be adequate to support the infant's need for maintaining blood pressure and modulating the inflammatory response. Concerns regarding the safety of the treatment of hydrocortisone are still evolving. In a large, randomized multicenter trial of hydrocortisone treatment in very low birth weight infants, an increase of spontaneous gastrointestinal perforations was found in the treated infants. ${ }^{8}$ As mentioned in the article by Efrid, this led to the halting of their study prior to complete enrollment. Even in the small number of infants enrolled in the study, there was a case of gastric perforation among the treated infants.

Additionally, little information is currently available regarding long-term outcome in infants who received hydrocortisone therapy. Physiologic stress doses of hydrocortisone are not the "supraphysiologic" doses used previously with dexamethasone that have been associated with poor neurodevelopmental outcome. In a neuronal cell culture model, stimulation of the glucocorticoid receptor, such as occurs with high-dose dexamethasone treatment, promotes apoptosis, whereas stimulation of the mineralocorticoid receptor, such as with low-dose therapy with hydrocortisone, is protective against apoptosis. ${ }^{9}$ Small studies have shown that hydrocortisone does not have the detrimental effects on growth that is seen with dexamethasone, and has not been associated with an increase in adverse neurodevelopmental outcome. $^{10}$
Hydrocortisone therapy has already become a useful tool in the neonatoligist's armamentarium of treatment of hypotension. The difficult question of who will benefit from hydrocortisone treatment and how to identify that subgroup of infants still remains. Low cortisol levels may aid in identifying this population, but the treatment of all infants with low cortisol levels is not warranted because of the ongoing potential concerns of both short- and longterm consequences of therapy. Additionally, high stress levels of cortisol may indicate a population more vulnerable to adverse outcomes where the addition of further hydrocortisone may be detrimental. Further investigation, especially regarding the longterm outcomes in infants exposed to hydrocortisone therapy, is needed before it is used as liberally as dexamethasone therapy was used in the past. Hydrocortisone therapy should be used judiciously, and length of treatment should be minimized in order to avoid excess exposure until further long-term safety is clarified.

\section{References}

1. Lambert SWJ, Bruining HA, deJong FH. Corticosteroid therapy in severe illness. N Engl J Med 1997;337:1285-92.

2. Watterberg KL. Adrenocortical function and dysfunction in the fetus and neonate. Semin Neonatol 2004;9:13-21.

3. Al Saedi S, Dean H, Dent W, Cronin C. Reference ranges for serum cortisol and 17-hydroxyprogesterone levels in preterm infants. J Pediatr 1995;126:985-7.

4. Seri I, Tan R, Evans J. Cardiovascular effects of hydrocortisone in preterm infants with pressor-resistant hypotension. Pediatrics 2001;107:1070-4.

5. Tantivit P, Subramanian N, Garg M, Ramanathan R, deLemos RA. Low serum cortisol in term infants with refactory hypotension. J Perinatol 1999;19:352-7.

6. Ng PC, Lee CH, Lam CW, et al. Transient adrenocortical insufficiency of prematurity and systemic hypotension in very low birth weight infants. Arch Dis Child Fetal Neonatal Ed 2004;89:F119-26.

7. Lago P, Benini F, Agosto C, Zacchello F. Randomised controlled trial of low dose fentanyl infusion in preterm infants with hyaline membrane disease. Arch Dis Child Fetal Neonatal Ed 1998;79:F194-7.

8. Watterberg KL, Gerdes J, Cole C, et al. Prophylaxis of early adrenal insufficiency to prevent bronchopulmonary dysplasia: multicenter trial. Pediatrics, in press.

9. Almeida OF, Conde GL, Crochemore C, et al. Subtle shifts in the ratio between pro- and antiapoptotic molecules after activation of corticosteroid receptors decide neuronal fate. FASEB J 2000;5:779-90.

10. van der Heide-Jalvin M, Kamphius PJ, van der Laan MJ, et al. Short- and long-term effects of neonatal glucocorticoid therapy: is hydrocortisone an alternative to dexamethasone? Acta Paediatr 2003;92:827-35. 